EPJ manuscript No.

(will be inserted by the editor)

\title{
Electroweak Physics at the Tevatron and LHC: Theoretical Status and Perspectives
}

\author{
Ulrich Baur \\ Physics Department, State University of New York at Buffalo, Buffalo, NY 14260, USA \\ Received: date / Revised version: date
}

\begin{abstract}
I review the status of theoretical calculations relevant for electroweak physics at the Tevatron and LHC and discuss future directions. I also give a brief overview of current electroweak data and discuss future expectations.
\end{abstract}

PACS. PACS-key describing text of that key - PACS-key describing text of that key

\section{Introduction}

Electroweak measurements are a very important part of the physics program of the Tevatron and the LHC. Of particular interest are the search for the Higgs boson and the determination of its properties, and the measurement of electroweak precision observables, in particular the measurement of

- the $W$ mass, $M_{W}$, and $W$ width, $\Gamma_{W}$,

- the effective weak mixing angle, $\sin ^{2} \theta_{\text {eff }}$, and the forward - backward asymmetry, $A_{F B}$,

- the $W$ and $Z$ boson cross sections, $\sigma(W)$ and $\sigma(Z)$, and their ratio, $R_{W / Z}$,

- the $W$ forward backward charge asymmetry, $A\left(\eta_{e}\right)$,

- the $\ell^{+} \ell^{-}(\ell \nu)$ differential cross sections above the $Z$ $(W)$ peak,

- and di-boson $(W \gamma, Z \gamma, W W, W Z$ and $Z Z)$ production.

In the following I discuss the physics interest in these measurements, give a brief overview of the current experimental status and what to expect in the future (for more details see Refs. [1 - 3]), and discuss the current status and the prospects of the relevant theoretical calculations.

\section{Weak Boson Physics}

\subsection{Measurement of the $W$ mass}

The one-loop corrections to $M_{W}$ depend quadratically on the top quark mass, $m_{t}$, and logarithmically on the Higgs boson mass, $m_{H}$. Precise measurements of $M_{W}$ and $m_{t}$ thus make it possible to extract information on $m_{H}$.

Send offprint requests to:
In Run I of the Tevatron, the $W$ mass has been measured to $M_{W}=80.456 \pm 0.059 \mathrm{GeV}$ 4. The preliminary value of the $W$ mass from LEP2 is $M_{W}=80.392 \pm$ $0.039 \mathrm{GeV}$ [5]. When combined with the current world average of the top quark mass, $m_{t}=172.7 \pm 2.9 \mathrm{GeV}$ [6], this yields $m_{H}<219 \mathrm{GeV}$ at $95 \% \mathrm{CL}$ [5] for a Standard Model (SM) Higgs boson.

In Run II, one hopes to achieve a precision of $\delta M_{W}=$ $40 \mathrm{MeV}$ per lepton channel and experiment for an integrated luminosity of $2 \mathrm{fb}^{-1}$ ], while the LHC may be able to reach a precision of $\delta M_{W} \approx 10 \mathrm{MeV}$ using the $W / Z$ transverse mass ratio and $W \rightarrow \mu \nu$ decays $[8$. The present constraints on $M_{W}$ and $m_{t}$ from LEP2 and Tevatron data, and the results expected from measurements at the LHC, are compared with theoretical predictions in Fig. 11 Present data clearly favor a light SM Higgs boson, and are also in very good agreement with predictions of the minimal supersymmetric standard model (MSSM) 9].

To ensure that the $M_{W}$ and $m_{t}$ measurements contribute equally to the uncertainty in a $\chi^{2}$ test, the precision on the top quark mass and the $W$ mass should satisfy the relation 10 .

$$
\delta M_{W} \approx 7 \times 10^{-3} \delta m_{t} .
$$

Since one expects to measure the top quark mass with a precision of $\delta m_{t}=1-2 \mathrm{GeV}$ at the LHC 11, one needs to determine the $W$ mass with a precision of about $\delta M_{W} \approx 10 \mathrm{MeV}$ so that it does not become the dominant uncertainty in the estimate of $m_{H}$. Accurate theoretical predictions for $W$ production are absolutely essential in order to measure the $W$ mass with a precision of $10 \mathrm{MeV}$.

\section{$2.2 \sin ^{2} \theta_{\text {eff }}$}

Constraints on $m_{H}$ can also be derived from the top quark mass and the effective weak mixing angle. At LEP, the ef- 


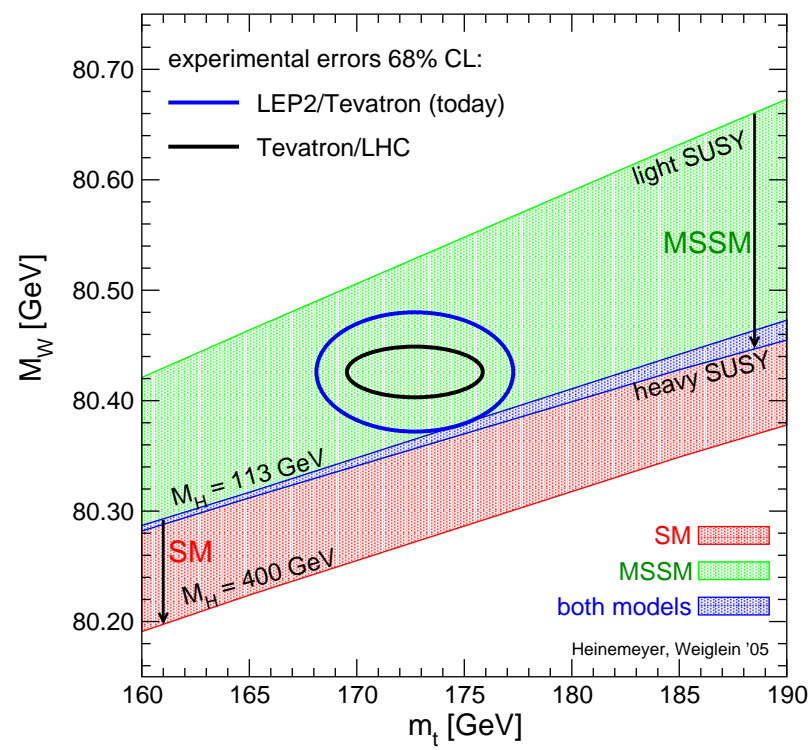

Fig. 1. Constraints on $M_{W}$ and $m_{t}$ from LEP2 and Tevatron data, and expectations from the LHC, compared with the predictions of the SM and the MSSM.

fective weak mixing angle has been measured to $\sin ^{2} \theta_{\text {eff }}=$ $0.23153 \pm 0.00016[12$. This will be difficult to improve at the Tevatron or LHC. From a measurement of the forward - backward asymmetry, $A_{F B}$, at the Tevatron one expects to reach a precision of $\delta \sin ^{2} \theta_{\text {eff }}=0.0006$ per lepton channel and experiment for an integrated luminosity of $10 \mathrm{fb}^{-1}$.7. At the LHC, with $100 \mathrm{fb}^{-1}$, one hopes to achieve $\delta \sin ^{2} \theta_{\text {eff }}=0.00014$ using forward electrons in a measurement of $A_{F B}$ in $Z \rightarrow e^{+} e^{-}$events [13].

\subsection{The Weak Boson Cross Sections and the $W / Z$ Cross Section Ratio}

In the past, the measurement of the $W$ and $Z$ boson cross sections has provided a test of perturbative QCD. With the large data sets of Run II and the LHC, non-statistical uncertainties, in particular the luminosity error, become limiting factors. This is illustrated by the recent $\mathrm{D} \varnothing$ measurements of the $W$ and $Z$ production cross sections [14]

$$
\begin{aligned}
\sigma(W) \cdot B(W \rightarrow e \nu)= & 2865.2 \pm 8.3(\text { stat }) \pm 62.8(\mathrm{sys}) \\
& \pm 40.4(\text { pdf }) \pm 186.2(\text { lumi }) \mathrm{pb}, \\
\sigma(Z) \cdot B\left(Z \rightarrow e^{+} e^{-}\right)= & 264.9 \pm 3.9(\text { stat }) \pm 8.5(\mathrm{sys}) \\
& \pm 5.1(\mathrm{pdf}) \pm 17.2 \text { (lumi) pb. }
\end{aligned}
$$

Provided that the $W$ and $Z$ cross sections are accurately predicted by theory, and the PDF uncertainties can be controlled, $\sigma(W)$ and $\sigma(Z)$ can be used as luminosity monitors.

The cross section ratio

$$
R_{W / Z}=\frac{\sigma(p \bar{p} \rightarrow W \rightarrow \ell \nu X)}{\sigma\left(p \bar{p} \rightarrow Z \rightarrow \ell^{+} \ell^{-} X\right)}
$$

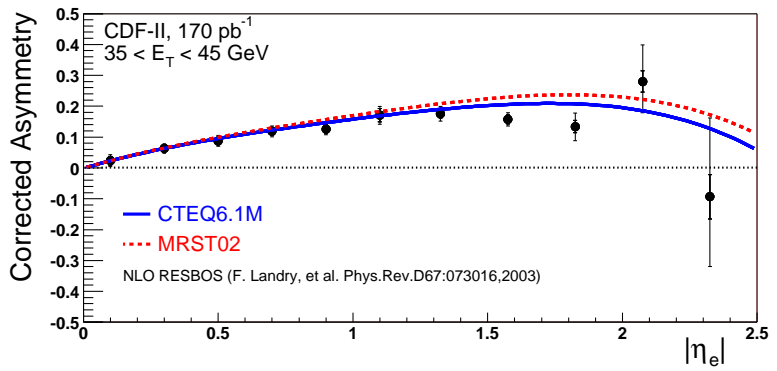

Fig. 2. The $W$ charge asymmetry as a function of $\eta_{e}$ for electrons with $35 \mathrm{GeV}<E_{T}<45 \mathrm{GeV}$ and two different PDF parametrizations.

together with the theoretical prediction for the ratio of the total $W$ and $Z$ production cross sections, the LEP measurement of the branching ratio $B\left(Z \rightarrow \ell^{+} \ell^{-}\right)$, and the SM prediction for the $W \rightarrow \ell \nu$ decay width, can be used for an indirect determination of $\Gamma_{W}$. A recent CDF measurement, $\Gamma_{W}=2079 \pm 41 \mathrm{MeV}$ [15], is in good agreement with the SM prediction $\Gamma_{W}^{S M}=2092 \pm 3 \mathrm{MeV}$ [16].

\subsection{Direct Measurement of $\Gamma_{W}$}

The width of the $W$ boson can also be measured directly from the tail of the $W \rightarrow \ell \nu(\ell=e, \mu)$ transverse mass $\left(M_{T}\right)$ distribution. Unlike the extraction of $\Gamma_{W}$ from $R_{W / Z}$, the measurement from the tail of the $M_{T}$ distribution does not depend on theoretical assumptions; however, the method is currently not as precise as the measurement using $R_{W / Z}$. This is illustrated by the recent combined Tevatron result, $\Gamma_{W}=2078 \pm 62$ (stat) \pm 60 (syst) $\mathrm{MeV}[17$. For $2 \mathrm{fb}^{-1}$ one expects the direct measurement of $\Gamma_{W}$ to improve to $\delta \Gamma_{W}=50 \mathrm{MeV}$ per lepton channel and experiment [18.

\subsection{The $W$ charge asymmetry}

Another important electroweak measurement is that of the $W$ charge asymmetry,

$$
A\left(\eta_{e}\right)=\frac{d \sigma\left(e^{+}\right) / d \eta_{e}-d \sigma\left(e^{-}\right) / d \eta_{e}}{d \sigma\left(e^{+}\right) / d \eta_{e}+d \sigma\left(e^{-}\right) / d \eta_{e}}
$$

where $\eta_{e}$ is the rapidity of the electron in $W \rightarrow e \nu . A\left(\eta_{e}\right)$ is sensitive to the $u$ - and $d$-quark components of the PDFs, especially at large values of $\eta_{e}$ and the electron transverse energy, $E_{T}$. Results from CDF for $170 \mathrm{pb}^{-1}$ of Run II data [19] are shown in Fig. 2]

\subsection{Search for New Physics in Drell-Yan Production}

Many models of new physics predict new charged $\left(W^{\prime}\right)$ or neutral $\left(Z^{\prime}\right)$ gauge bosons. One can search for these particles in the high $\ell^{+} \ell^{-}(\ell \nu)$ invariant (transverse) mass region. Information on the couplings of a $Z^{\prime}$ boson can be 
obtained from the forward - backward asymmetry, $A_{F B}$, at large di-lepton masses. Present $\mathrm{D} \varnothing$ data $\left(200 \mathrm{pb}^{-1}\right)$ require that $m_{Z^{\prime}}>780 \mathrm{GeV}$ at $95 \% \mathrm{CL}$ for a SM-like $Z^{\prime}$ boson 20].

At the LHC, one can discover a $Z^{\prime}$ boson with $m_{Z^{\prime}}=$ $4-5 \mathrm{TeV}$ for $300 \mathrm{fb}^{-1}$ and one will be able to severely constrain the couplings of the new vector boson [21].

\subsection{Theory of Single Weak Boson Production}

The precision foreseen for electroweak measurements in Run II and at the LHC has to be matched by precise theoretical predictions, ie. QCD and electroweak (EW) radiative corrections have to be under control.

The QCD corrections to the total $W$ and $Z$ boson cross sections at the next-to-next-to-leading (NNLO) level have been known for more than a decade [22. Recently, the rapidity distribution of the $Z$ boson has been calculated at NNLO, showing a dramatically reduced dependence of the differential cross section on the unphysical renormalization and factorization scales compared with the NLO prediction 23. Calculations of the resummed QCD corrections to predict the transverse momentum $\left(q_{T}\right)$ distributions of the $W$ and $Z$ bosons are also available [24]. The precise shape of the weak boson $q_{T}$ distribution for small transverse momenta, however, is still uncertain, in particular at the LHC [25].

With the the uncertainty from unknown higher order QCD corrections approaching the 1\% level [23, EW radiative corrections to weak boson production become important. EW corrections may also be enhanced by collinear logarithms near the $W$ and $Z$ resonances, and by Sudakov logarithms at large $\ell^{+} \ell^{-}$and $\ell \nu$ invariant masses. A consistent calculation of $\mathrm{EW}$ radiative corrections requires parton distribution functions (PDFs) which take into account QED corrections. Such PDFs exist now [26].

There has been significant progress in the calculation of the EW radiative corrections to $W$ and $Z$ boson production in the past few years. Calculations of the full $\mathcal{O}(\alpha)$ EW corrections are available now [27,28].

The main effect of the EW corrections in the vicinity of the $W$ and $Z$ resonances is that they shift the $W$ and $Z$ masses extracted from data. The magnitude of the shift is about $50 \mathrm{MeV}(150 \mathrm{MeV})$ for $W \rightarrow e \nu$ $(W \rightarrow \mu \nu)$. Since both leptons can radiate photons, the shifts are about twice as large in $Z$ events. The shift is mostly caused by final state photon radiation which is enhanced due to collinear logarithms of the form $(\alpha / \pi)$ $\log \left(M_{W, Z}^{2} / m_{\ell}^{2}\right)$, where $m_{\ell}$ is the mass of the charged lepton in $W \rightarrow \ell \nu$ or $Z \rightarrow \ell^{+} \ell^{-}$. Final state photon radiation distorts the shape of the Breit-Wigner resonance by reducing the peak cross section by $20-30 \%$, and (significantly) enhancing the cross section below the peak. This is shown in Fig. 3 for the $Z$ case with only QED corrections taken into account 29.

Above the $W / Z$ peak, the purely weak corrections become increasingly important, due to Sudakov-like logarithms of the form $(\alpha / \pi) \log ^{2}\left(\hat{s} / M_{W, Z}^{2}\right)$, where $\hat{s}$ is the

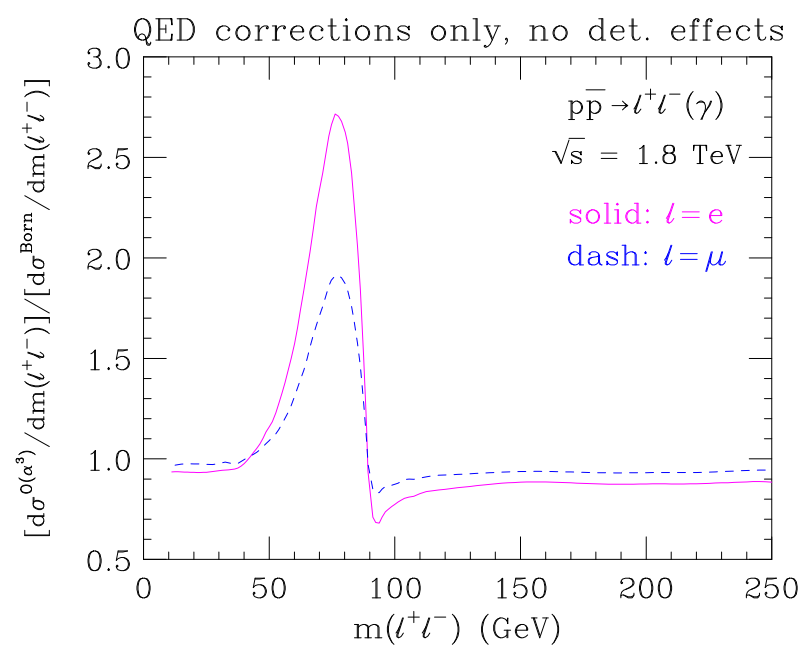

Fig. 3. Ratio of the $\mathcal{O}\left(\alpha^{3}\right)$ and lowest order differential cross sections as a function of the di-lepton invariant mass for $p \bar{p} \rightarrow$ $\ell^{+} \ell^{-}(\gamma)$ at $\sqrt{s}=1.8 \mathrm{TeV}$

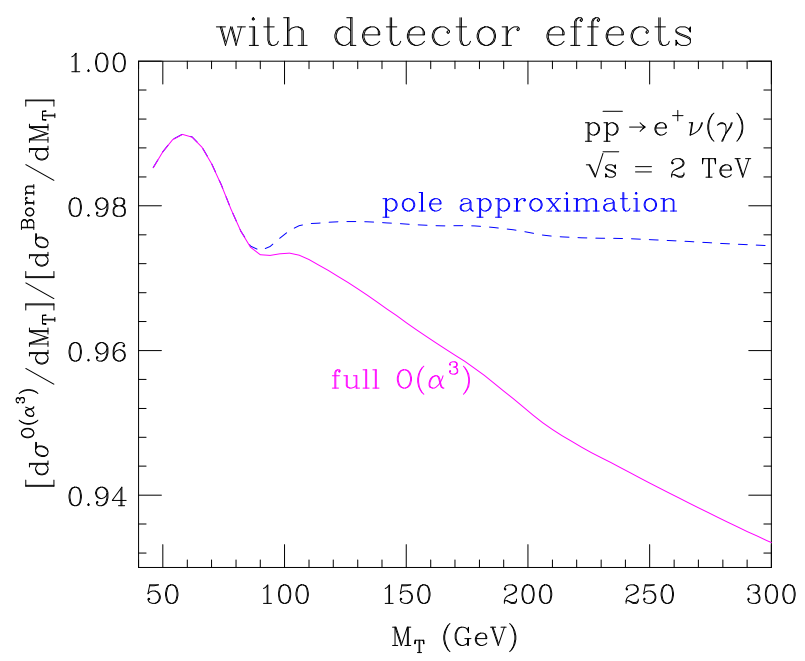

Fig. 4. The ratio $\left[d \sigma^{\mathcal{O}\left(\alpha^{3}\right)} / d M_{T}\right] /\left[d \sigma^{\text {Born }} / d M_{T}\right]$ as a function of the transverse mass for $p \bar{p} \rightarrow e^{+} \nu_{e}(\gamma)$ at $\sqrt{s}=2 \mathrm{TeV}$.

squared invariant mass of the $\ell^{+} \ell^{-}$or $\ell \nu$ system. This is shown in Fig. 4 for the $e \nu$ transverse mass. The solid line shows the ratio $\left[d \sigma^{\mathcal{O}\left(\alpha^{3}\right)} / d M_{T}\right] /\left[d \sigma^{\text {Born }} / d M_{T}\right]$ taking into account the complete $\mathcal{O}(\alpha)$ EW corrections. The dashed line shows the ratio in the pole approximation 27. 28 where the $W Z$ box diagrams responsible for the $\mathrm{Su}-$ dakov logarithms are absent.

Since the logarithmic terms from the $W Z$ box diagrams change the slope of the $M_{T}$ distribution, they shift the $W$ width extracted from the tail of the $M_{T}$ distribution. This shift, $\delta \Gamma_{W} \approx-7 \mathrm{MeV}$ [27, while not large, cannot be neglected if the Run II goal (see Sec. 2.4) should be met.

At the LHC, it will be possible to probe di-lepton invariant and $\ell \nu$ transverse masses of several $\mathrm{TeV}$. In this region, the Sudakov logarithmic terms grow so large that 
they have to be resummed. Although the resummation of electroweak Sudakov-like logarithms in general four fermion electroweak processes has been discussed in the literature 30 31, a calculation of $\ell \nu$ production in hadronic collisions which includes resummation of electroweak logarithms has not been carried out yet.

Since final state photon radiation causes a significant shift in $M_{W}$ and $M_{Z}$, one has to worry about multiple (final) state photon radiation in weak boson production. Two photon radiation is known to considerably change the shape of the dilepton and $\ell \nu$ transverse mass distributions 32. Recently, there have been several calculations of multi-photon radiation in $W$ 33,34 and $Z$ decays 35. The shift in the weak boson masses caused by multiple photon radiation is found to be about $10 \%$ of the shift originating from one-photon emission 34,35. For the muon final state, where the shift in the weak boson masses is particularly large, this is a non-negligible effect.

The experimental precision which can be achieved for $M_{W}$ strongly depends on how well the transverse momentum distribution of the $W$ is known. Knowledge of the $W q_{T}$ distribution determines the missing transverse energy $\left(\mathscr{E}_{T}\right)$ resolution in $W$ events. The $\mathscr{E}_{T}$ resolution determines how "sharp" the edge in the $M_{T}$ distribution at $M_{T} \approx M_{W}$ is, which in turn determines how well $M_{W}$ can be measured. To constrain the $W q_{T}$ distribution, one uses data on the transverse momentum distribution of the $Z$ boson, together with a theoretical prediction for the ratio $\left[d \sigma(W) / d q_{T}(W)\right] /\left[d \sigma(Z) / d q_{T}(Z)\right]$. For the $W$ mass measurement one thus needs a calculation which includes both the resummed QCD corrections, the full $\mathcal{O}(\alpha)$ EW corrections, and effects from multiple photon radiation. A first step towards this lofty goal has been taken in Ref. [36, where final state photon radiation was added to a calculation of $W$ boson production which includes resummed QCD corrections.

\section{Di-boson Production}

\subsection{Experimental Results}

Di-boson production makes it possible to probe the $W W \gamma$, $W W Z, Z \gamma \gamma, Z Z \gamma$ and $Z Z Z$ self-couplings (TGCs). For details on these couplings and recent TGC measurements at the Tevatron see Ref. 3. In the following I concentrate on the $W W \gamma$ and $W W Z$ couplings and briefly summarize recent experimental results for these couplings.

The most general $W W V(V=\gamma, Z)$ vertex consistent with Lorentz invariance and electromagnetic gauge invariance has seven free parameters 37 . Assuming $C$ and $P$ conservation, five independent couplings, $g_{1}^{Z}, \kappa_{V}$ and $\lambda_{V}$, remain. Requiring $\mathrm{SU}(2)$ invariance as well, $\lambda_{Z}=\lambda_{\gamma}$ and $\kappa_{Z}=g_{1}^{Z}-\left(\kappa_{\gamma}-1\right) \tan ^{2} \theta_{W}$, where $\theta_{W}$ is the weak mixing angle [38, and one is left with three independent couplings. In the SM, at tree level, $g_{1}^{Z}=\kappa_{V}=1$ and $\lambda_{V}=0$. In order to avoid that $S$-matrix unitarity is violated, deviations of the TGCs from their SM values have to be momentum dependent form factors which depend on the form factor scale, $\Lambda$ 39. The form factor scale is related to the scale of the new physics which causes non-SM TGCs.

The $W W V$ couplings can be measured in $e^{+} e^{-} \rightarrow$ $W^{+} W^{-}$, and in $W \gamma, W Z$ and $W W$ pair production at hadron colliders. Assuming $C, P$ and $\mathrm{SU}(2)$ invariance, the LEP experiments have determined the independent couplings to 40]

$$
\begin{aligned}
& g_{1}^{Z}=0.984_{-0.019}^{+0.022}, \\
& \kappa_{\gamma}=0.973_{-0.045}^{+0.044}, \\
& \lambda_{\gamma}=-0.028_{-0.021}^{+0.020} .
\end{aligned}
$$

$W^{+} W^{-}$production is sensitive to both the $W W \gamma$ and the $W W Z$ couplings. To measure these couplings independently, one has to consider $W \gamma$ and $W Z$ production in hadronic collisions. Measurements of the $W W \gamma$ couplings in $W \gamma$ production have been performed in Run I 41 and in Run II [3]. The D $\varnothing$ Collaboration recently presented the first direct measurement of the $W W Z$ couplings from $W Z$ production. For $0.3 \mathrm{fb}^{-1}$, and assuming $\Lambda=1.5 \mathrm{TeV}$, they found that 42

$$
\begin{aligned}
-0.48<\lambda_{Z}<0.48 & \text { for } \kappa_{Z}=g_{1}^{Z}=1, \\
0.51<g_{1}^{Z}<1.66 & \text { for } \lambda_{Z}=\kappa_{Z}-1=0
\end{aligned}
$$

at $95 \%$ CL. Note that, at hadron colliders, TGC limits depend on the form factor scale, $\Lambda$.

Bounds on TGCs from hadron collider experiments scale roughly with $\left(\int \mathcal{L} d t\right)^{1 / 4}$. One thus expects that the ultimate precision which can be reached for the $W W V$ couplings at the Tevatron will be a factor 1.6 to 2.5 better than that obtained from current data, depending on the final integrated luminosity. While the TGC bounds at the Tevatron only mildly depend on the form factor scale, the dependence on $\Lambda$ is much more pronounced at the LHC. In general, the $W W V$ couplings can be measured with a precision of $\mathcal{O}\left(10^{-2}-10^{-3}\right)$ at the LHC [10].

\subsection{Theory of Di-boson Production}

All di-boson production processes are known to NLO in QCD 43 . At the LHC QCD corrections to di-boson production are large and increase with the $p_{T}$ of the vector bosons. The ratio of the NLO to LO cross sections ( $k$ factor) for $W^{+} Z$ production at the LHC as a function of $p_{T}(Z)$ is shown in Fig. 5 Qualitatively similar results are obtained for the other di-boson processes. Since the QCD corrections give an effect which is qualitatively similar to that of anomalous TGCs, it will be essential to take them into account in any LHC di-boson analysis.

The EW radiative corrections to the di-boson production processes are also known 44. As in the case of single weak boson production, they become significant at large energies, due to EW Sudakov logarithms. For invariant masses in the $\mathrm{TeV}$ region they reduce the cross section by typically $5-20 \%$. 


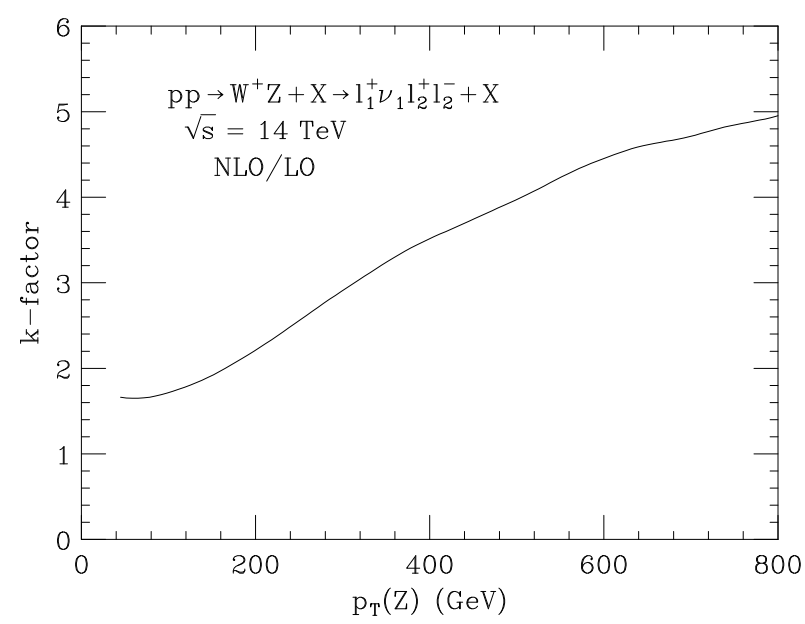

Fig. 5. The ratio of the NLO to LO cross sections as a function of $p_{T}(Z)$ for $W^{+} Z$ production at the LHC.

\section{Higgs Boson Physics}

The search for the SM Higgs boson is one of the main objectives of the LHC. Over the last decade, enormous progress has been made in providing accurate predictions for Higgs boson production and decays. In addition, in the last few years, many studies of how well the Higgs properties can be determined once this particle has been found have been performed.

The NLO QCD corrections to Higgs production via gluon fusion have been calculated more than 10 years ago 45. They enhance the $g g \rightarrow H$ cross section by a factor $1.5-2$. More recently, several groups have calculated the NNLO QCD corrections to the total $g g \rightarrow H$ cross section in the $m_{t} \rightarrow \infty$ limit 46, showing that the perturbative series starts to converge at this order. A fully differential NNLO calculation for $g g \rightarrow H \rightarrow \gamma \gamma$ also exists 47. Finally, the $\mathcal{O}(\alpha)$ corrections to Higgs production via gluon fusion have been computed 48. They change the Higgs production cross section by $5-8 \%$ if $m_{H}=115-160 \mathrm{GeV}$.

For $m_{H}<200 \mathrm{GeV}$, production via vector boson fusion (VBF), $q q^{\prime} \rightarrow H q q^{\prime}$, is an important source for Higgs bosons. The QCD corrections to $q q^{\prime} \rightarrow H q q^{\prime}$ have been found to be modest 49. Associated production of Higgs bosons and top quarks, $p p \rightarrow t \bar{t} H$, is a tool for measuring the top quark Yukawa coupling. At LO, the $t \bar{t} H$ cross section strongly depends on the factorization and renormalization scales. Once NLO QCD corrections are taken into account, this dependence is greatly reduced [50].

While Higgs production is well under control theoretically, more reliable calculations are still needed for several background processes. In particular, calculations of the NLO QCD corrections are needed for $t \bar{t} j, t \bar{t} b \bar{b}$ and EW $W W j j$ production.

Once a Higgs candidate has been found, one would like to determine how the new particle couples to fermions, gauge bosons, and to itself. Several studies have shown that, with mild theoretical assumptions, the couplings of

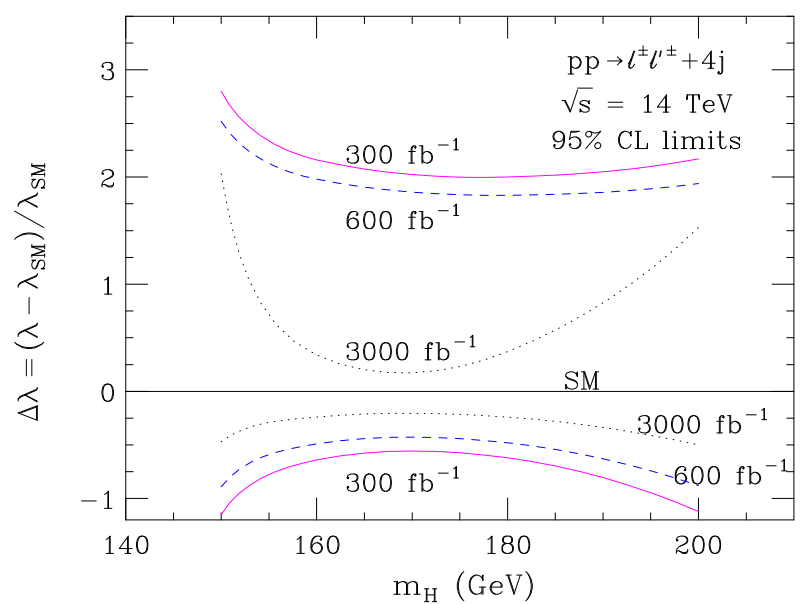

Fig. 6. Limits achievable at $95 \%$ CL for the normalized Higgs boson self-coupling, $\Delta \lambda_{H H H}=\left(\lambda-\lambda_{S M}\right) / \lambda_{S M}$, at the LHC.

the new particle to fermions and gauge bosons can be measured with a precision of $10-30 \%$ at the LHC 51 .

A measurement of the three Higgs boson self-coupling, $\lambda$, with a similar precision is considerably more difficult. In order to probe $\lambda$, one has to study Higgs pair production, $g g \rightarrow H H$. For $m_{H} \geq 150 \mathrm{GeV}$, the $H H \rightarrow 4 W \rightarrow$ $\ell^{ \pm} \ell^{\prime \pm}+4 \mathrm{j}$ channel offers the best chances $[52,53$. As shown in Fig. 6] with $300 \mathrm{fb}^{-1}$, it may be possible to rule out a vanishing of $\lambda$ for $m_{H}=150-200 \mathrm{GeV}$, and measure the $H H H$ coupling with up to $20 \%$ accuracy at a SuperLHC with $3 \mathrm{ab}^{-1}$. For $m_{H} \leq 140 \mathrm{GeV}, H H \rightarrow b \bar{b} \gamma \gamma$ is the most promising final state. However, due to the tiny signal cross section in this channel, a luminosity upgrade for the LHC is needed. Even with $3 \mathrm{ab}^{-1}$ one can only hope to achieve a precision of about $70 \%$ for $\lambda[54$.

While the signal to background ratio is of $\mathcal{O}(1)$ for $H H \rightarrow b \bar{b} \gamma \gamma$, it is roughly $1 / 5-1 / 10$ for the $\ell^{ \pm} \ell^{\prime \pm}+4 \mathrm{j}$ final state. The largest backgrounds contributing to $\ell^{ \pm} \ell^{\prime \pm}+4 \mathrm{j}$ production originate from $W W W j j, t \bar{t} W$ and $t \bar{t} j$ production. The $t \bar{t} j$ background is particularly sensitive to the acceptance cuts imposed, and thus tricky to estimate. More realistic simulations for this background are needed. Furthermore, both signal and background cross sections show a significant scale dependence which could be reduced by full calculations of the NLO QCD corrections to $g g \rightarrow H H$ (for finite $m_{t}$ ), and the background reactions. None of these exist at the moment.

\section{Summary}

Electroweak physics at hadron colliders is precision physics. Accurate predictions are needed to fully utilize the potential of the Tevatron and LHC for electroweak measurements. The theoretical predictions for weak boson and Higgs boson production have become increasingly accurate over the past few years. However, there is still much to do. In particular a calculation which combines QCD and EW radiative corrections for $W$ and $Z$ is needed, as well 
as calculations of the NLO QCD corrections to a number of processes which contribute to the background in Higgs production.

\section{Acknowledgments}

This research was supported by the National Science Foundation under grant No. PHY-0139953.

\section{References}

1. S. Protopopescu, these proceedings.

2. M. Lancaster, these proceedings.

3. A. Goshaw, these proceedings.

4. V. M. Abazov et al. [CDF and D $\varnothing$ Collaborations], Phys. Rev. D 70 (2004) 092008.

5. M. Grünewald, talk given at the EPS2005 conference, Lisbon, Portugal, July 2005; C. Diaconu, arXiv:hep-ex/0510035

6. The CDF and DØ Collaborations, arXiv:hep-ex/0507091

7. R. Brock et al., arXiv:hep-ex/0011009

8. A. Schmidt, Diplomarbeit, Universität Karlsruhe, Germany, 2004; F. Gianotti, ATL-PHYS-99-001.

9. S. Heinemeyer and G. Weiglein, arXiv:hep-ph/0012364 A. Djouadi et al., Phys. Rev. Lett. 78 (1997) 3626; Phys. Rev. D 57 (1998) 4179; S. Heinemeyer and G. Weiglein, JHEP 0210 (2002) 072.

10. S. Haywood et al., arXiv:hep-ph/0003275

11. M. Beneke et al., arXiv:hep-ph/0003033

12. S. Schael et al. [The ALEPH, DELPHI, L3, OPAL and SLD Collaborations], arXiv:hep-ex/0509008

13. K. Sliwa, S. Riley, and U. Baur, ATL-PHYS-2000-018.

14. The DØ Collaboration, DØnote 4403-Conf (2004).

15. A. Abulencia et al. [CDF Collaboration], arXiv:hep-ex/0508029

16. K. Hagiwara et al. [Particle Data Group], Phys. Rev. D 66 (2002) 010001.

17. B. Ashmankas et al. [Tevatron Electroweak Working Group], arXiv:hep-ex/0510077

18. D. Amidei et al. [TeV-2000 Study Group], SLACREPRINT-1996-085.

19. D. Acosta et al. [CDF Collaboration], Phys. Rev. D 71 (2005) 051104.

20. The DØ Collaboration, DØnote 4375-CONF (2004).

21. See http://cmsdoc.cern.ch/cms/PRS/results/susybsm/ susybsm.html.

22. W. L. van Neerven and E. B. Zijlstra, Nucl. Phys. B 382 (1992) 11 [Erratum-ibid. B 680 (2004) 513].

23. C. Anastasiou et al., Phys. Rev. Lett. 91 (2003) 182002; Phys. Rev. D 69 (2004) 094008.

24. C. Balazs, J. W. Qiu and C. P. Yuan, Phys. Lett. B 355 (1995) 548.

25. S. Berge et al., Phys. Rev. D 72 (2005) 033015.

26. A. D. Martin et al., Eur. Phys. J. C 39 (2005) 155.

27. U. Baur et al., Phys. Rev. D 65 (2002) 033007.

28. S. Dittmaier and M. Krämer, Phys. Rev. D 65 (2002) 073007; U. Baur and D. Wackeroth, Phys. Rev. D 70 (2004) 073015.

29. U. Baur, S. Keller and W. K. Sakumoto, Phys. Rev. D 57 (1998) 199.
30. P. Ciafaloni and D. Comelli, Phys. Lett. B446 (1999) 278.

31. J. H. Kühn et al., Nucl. Phys. B 616 (2001) 286 [Erratumibid. B 648 (2003) 455]; J. H. Kühn, A. A. Penin and V. A. Smirnov, Eur. Phys. J. C 17 (2000) 97; M. Beccaria et al., Phys. Rev. D 61 (2000) 073005; J. H. Kühn and A. A. Penin, arXiv:hep-ph/9906545

32. U. Baur and T. Stelzer, Phys. Rev. D 61 (2000) 073007.

33. W. Placzek and S. Jadach, Eur. Phys. J. C 29 (2003) 325.

34. C. M. Carloni Calame et al., Eur. Phys. J. C 33 (2004) S665.

35. C. M. Carloni Calame et al., JHEP 0505 (2005) 019.

36. Q. H. Cao and C. P. Yuan, Phys. Rev. Lett. 93 (2004) 042001.

37. K. Hagiwara et al., Nucl. Phys. B 282 (1987) 253.

38. K. Hagiwara et al., Phys. Lett. B 283 (1992) 353; Phys. Rev. D 48 (1993) 2182.

39. U. Baur and D. Zeppenfeld, Phys. Lett. B 201 (1988) 383.

40. The LEP Collaborations, LEPEWWG/TGC/2005-01.

41. See J. Ellison and J. Wudka, Ann. Rev. Nucl. Part. Sci. 48 (1998) 33 and references therein.

42. V. M. Abazov et al. [D $\varnothing$ Collaboration], Phys. Rev. Lett. 95 (2005) 141802.

43. J. Ohnemus and J. F. Owens, Phys. Rev. D 43 (1991) 3626; J. Ohnemus, Phys. Rev. D 44 (1991) 1403; Phys. Rev. D 44 (1991) 3477; Phys. Rev. D 47 (1993) 940; U. Baur, T. Han and J. Ohnemus, Phys. Rev. D 48 (1993) 5140; Phys. Rev. D 51 (1995) 3381; Phys. Rev. D 53 (1996) 1098; Phys. Rev. D 57 (1998) 2823; J. M. Campbell and R. K. Ellis, Phys. Rev. D 60 (1999) 113006; L. J. Dixon, Z. Kunszt and A. Signer, Nucl. Phys. B 531 (1998) 3; Phys. Rev. D 60 (1999) 114037; K. L. Adamson, D. de Florian and A. Signer, Phys. Rev. D 65 (2002) 094041; Phys. Rev. D 67 (2003) 034016.

44. E. Accomando, A. Denner and S. Pozzorini, Phys. Rev. D 65 (2002) 073003; E. Accomando, A. Denner and A. Kaiser, Nucl. Phys. B 706 (2005) 325; E. Accomando, A. Denner and C. Meier, arXiv:hep-ph/0509234 W. Hollik and C. Meier, Phys. Lett. B 590 (2004) 69.

45. M. Spira et al., Nucl. Phys. B 453 (1995) 17.

46. R. V. Harlander and W. B. Kilgore, Phys. Rev. Lett. 88 (2002) 201801; C. Anastasiou and K. Melnikov, Nucl. Phys. B 646 (2002) 220; V. Ravindran, J. Smith and W. L. van Neerven, Nucl. Phys. B 665 (2003) 325.

47. C. Anastasiou, K. Melnikov and F. Petriello, arXiv:hep-ph/0501130

48. U. Aglietti et al., Phys. Lett. B 600 (2004) 57; G. Degrassi and F. Maltoni, Phys. Lett. B 600 (2004) 255.

49. T. Han, G. Valencia and S. Willenbrock, Phys. Rev. Lett. 69 (1992) 3274; T. Figy, C. Oleari and D. Zeppenfeld, Phys. Rev. D 68 (2003) 073005; E. L. Berger and J. Campbell, Phys. Rev. D 70 (2004) 073011.

50. W. Beenakker et al., Nucl. Phys. B 653 (2003) 151; L. Reina, S. Dawson and D. Wackeroth, Phys. Rev. D 65 (2002) 053017; S. Dawson et al., Phys. Rev. D 67 (2003) 071503; S. Dawson et al., Phys. Rev. D 68 (2003) 034022.

51. M. Dührssen et al., Phys. Rev. D 70 (2004) 113009 and references therein.

52. F. Gianotti et al., Eur. Phys. J. C 39 (2005) 293.

53. U. Baur, T. Plehn and D. L. Rainwater, Phys. Rev. Lett. 89 (2002) 151801; Phys. Rev. D 67 (2003) 033003; Phys. Rev. D 68 (2003) 033001.

54. U. Baur, T. Plehn and D. L. Rainwater, Phys. Rev. D 69 (2004) 053004. 